PAPERS

\title{
Nanoparticle shapes: Quantification by elongation, convexity and circularity measures
}

\author{
Lazar Kopanja $^{* * *}$, Boris Lončar* ${ }^{*}$ Dragiša Žunić ${ }^{* * *}$, Marin Tadićc*** $^{* * * *}$
}

\begin{abstract}
The goal of the nanoparticle synthesis is, first of all, the production of nanoparticles that will be more similar in size and shape. This is very important for the possibility of studying and applying nanomaterials because of their characteristics that are very sensitive to size and shape such as, for example, magnetic properties. In this paper, we propose the shape analysis of the nanoparticles using three shape descriptors - elongation, convexity and circularity. Experimental results were obtained by using TEM images of hematite nanoparticles that were, first of all, subjected to segmentation in order to obtain isolated nanoparticles, and then the values of elongation, convexity and circularity were measured. Convexity $C_{\mathrm{x}}(S)$ is regarded as the ratio between shape's area and area of the its convex hull. The convexity measure defines the degree to which a shape differs from a convex shape while the circularity measure defines the degree to which a shape differs from an ideal circle. The range of convexity and circularity values is $(0,1]$, while the range of elongation values is $[1, \infty)$. The circle has lowest elongation $(\varepsilon=1)$, while it has biggest convexity and circularity values $\left(C_{\mathrm{x}}=1 ; C=1\right)$. The measures $\varepsilon(S), C_{\mathrm{x}}(S), C(S)$ proposed and used in the experiment have the few desirable properties and give intuitively expected results. None of the measures is good enough to describe all the shapes, and therefore it is suggested to use a variety of measures so that the shapes can be described better and then classify and control during the synthesis process.
\end{abstract}

K e y w or ds: shape analysis, nanoparticles, elongation, shape descriptors, circularity, convexity

\section{Introduction}

Nanotechnologies offer many possibilities for the development of new materials with improved properties for applications in electronics, optoelectronics, chemical engineering, mechanical engineering, microbiological, biomedicine, etc. It is well known that the size and shape of the nanoparticles significantly influence the magnetic properties of nanomaterials and that, by their adjustment, the magnetic properties can be controlled in a wide range [1-3]. However, the area of magnetic nanomaterials is still an insufficiently developed area, and one of the causes is that the synthesized nanoparticles differ in shape and size, so that their influence on magnetic properties cannot be clearly seen. The main goal in nanoparticle synthesis is to obtain the desired material with a well-defined shape and size of nanoparticles (narrow distribution of particles by size and shape).

By reducing the size of the magnetic particles, magnetic properties change, so magnetic nanomaterials exhibit different physical properties: high coercivity, superparamagnetism, high magnetic resistance, low or high magnetization, a decrease in the Curie/Neel temperature. One of the very important anisotropy of the nanoparticles that determines the magnetic properties of the nanomaterials is a shape anisotropy, which is directly related to the nanoparticle shape. The values of the magnetic parameters can differ in the same materials even ten times depending on the shape of the nanoparticles [4-7]. Recently, the influence of nanoparticles on the macroscopic magnetic properties of nanomaterials is increasingly being studied in literature [8-10]. For example, the shape elongation plays an important role in understanding the magnetic properties of nanomaterials. It is known that coercivity increases with increasing elongation $[6,11]$. Due to this influence of the shape on the physical properties of the materials, in addition to measuring the physical properties, it is very important to have a procedure to measure the shape, and assign to a shape the quantitative values based on what the nanoparticles can be compared and classified.

The starting point of the algorithm for measuring the shape are microscopic images of nanomaterials that are then analyzed in order to determine the quantitative characteristics of the nanoparticles and their mutual relations. In image analysis procedures image segmentation is one of the first steps [12-14]. Image segmentation is the process of dividing an image into its constituent segments until to the required level. Segments are defined differently depending on the need for which the image is analyzed. In the case of nanomaterial images, the goal of the segmen-

*Faculty of Technology and Metallurgy, University of Belgrade, Karnegijeva 4, PO Box 3503, 11120 Belgrade, Serbia, **Faculty of Mathematics and Computer Science, Alfa BK University, Palmira Toljatija 3, 11070 Belgrade, Serbia, ***Carnegie Mellon University in Qatar, Education City, P.O. Box 24866, Doha, Qatar, ****Condensed Matter Physics Laboratory, Vinca Institute, University of Belgrade, P.O. Box 522, 11001 Belgrade, Serbia, kopanjal@yahoo.com

DOI: $10.2478 /$ jee-2019-0040, Print (till 2015) ISSN 1335-3632, On-line ISSN 1339-309X

(C) This is an open access article licensed under the Creative Commons Attribution-NonCommercial-NoDerivs License (http://creativecommons.org/licenses/by-nc-nd/3.0/) 
tation is to obtain separated nanoparticles whose shape is analyzed in the next phase.

For this analysis, a mathematical tool is needed to describe the shapes quantitatively. For this purpose, a number of shape descriptors are developed to answer the question of how much a shape is similar to the corresponding observed shape, such as a circle (circularity), a square (squareness), an ellipse (ellipticity), a triangle (triangularity), a rectangle (rectangularity) [8, 15-27]. In order to be useful, each of the shape descriptors should ensure that the appropriate measure is invariant with respect to translation, rotation and scaling. In addition, it is important that there is a fast, efficient and easily understandable procedure for calculating these measures.

In this paper, for the determination of the morphological characteristics of the nanoparticles, the use of three descriptors is proposed: elongation, convexity and circularity. The elongation measure $\varepsilon(S)$ gives an answer to the question of how much nanoparticle shape have been elongated. The smallest elongation has a circle, which has a measure of elongation equal to 1 . Convexity $C_{\mathrm{x}}(S)$ is defined as the ratio between shape's area and area of the its convex hull [28]. The convexity measure defines the degree to which a shape differs from a convex shape. For all shapes, the values of convexity measure are in the interval $(0,1]$. Only in the case of a convex shape, the value of the convexity measure is equal to 1 . On the other hand, circularity $C(S)$ represents the degree of deviation of the shape from the ideal circle. As with convexity, for all shapes the values of circularity measure are in the interval $(0,1]$. Only in the case of a circle, the value of the circularity measure is equal to 1 .

Also, three measures $\varepsilon(S), C_{\mathrm{x}}(S)$ and $C(S)$ proposed and used in the experiment gives intuitively expected results. These measures are clearly defined, relatively easy to use and there is a fast and easily understood procedure for their computing. Next to that, the proposed measures are invariant with respect to similarity transformations (translation, rotation and scaling). In order to calculate the measures of elongation, convexity and circularity, the MATLAB software package is used, where algorithms for calculating these shape descriptors are implemented.

\section{Experiment}

This section outlines the basic characteristics and theoretical bases of the proposed measures.

\subsection{Elongation measure}

The elongation measure is often considered in literature as one of the basic shape descriptors. The shape elongation is closely linked to the shape orientation. The standard approach to calculating the shape orientation defines the orientation using the so-called minimal moment axis of the second order [8, 20, 29-30]. It is a line that minimizes the integral of the square distances from the points that belong to the shape to that line. The integral is

$$
I(S, \varphi, \rho)=\iint_{S} r^{2}(x, y, \varphi, \rho) \mathrm{d} x \mathrm{~d} y
$$

where $r(x, y, \varphi, \rho)$ is the distance from the $(x, y)$ to the line

$$
x \cos \varphi-y \sin \varphi=\rho .
$$

The line that minimizes the integral $I(S, \varphi, \rho)$ passes through the centroid $\left(x_{\mathrm{c}}(S), y_{\mathrm{c}}(S)\right)$ of the shape $S$ where

$$
\left(x_{\mathrm{c}}(S), y_{\mathrm{c}}(S)\right)=\left(\frac{\iint_{S} x \mathrm{~d} x \mathrm{~d} y}{\iint_{S} \mathrm{~d} x \mathrm{~d} y}, \frac{\iint_{S} y \mathrm{~d} x \mathrm{~d} y}{\iint_{S} \mathrm{~d} x \mathrm{~d} y}\right) .
$$

Without loss of generality, it can be assumed that the origin is placed at the centroid, ie $\left(x_{\mathrm{c}}(S), y_{\mathrm{c}}(S)\right)=(0,0)$. Considering that the required line that minimizes the integral $I(S, \varphi, \rho)$ passes through the origin, it can be put $\rho=0$. The square of the distance the point $(x, y)$ to the line $x \cos \varphi-y \sin \varphi=\rho$ is equal $(x \sin \varphi-y \cos \varphi)^{2}$, thus, the shape orientation problem can be reformulated to the problem of determining $\varphi$ for which $I(\varphi, S)$ defined as

$$
I(\varphi, S)=I(S, \varphi, \rho=0)=\iint_{S}(x \sin \varphi-y \cos \varphi)^{2} \mathrm{~d} x \mathrm{~d} y
$$

reaches the minimum. The function $I(\varphi, S)$ can be written in the form

$$
\begin{aligned}
I(\varphi, S)= & \sin ^{2} \varphi \iint_{S} x^{2} \mathrm{~d} x \mathrm{~d} y-\sin 2 \varphi \iint_{S} x y \mathrm{~d} x \mathrm{~d} y+ \\
& \cos ^{2} \varphi \iint_{S} y^{2} \mathrm{~d} x \mathrm{~d} y .
\end{aligned}
$$

If the central geometric moments $\bar{m}_{\mathrm{p}, \mathrm{q}}(S)$ are defined as

$$
\bar{m}_{\mathrm{p}, \mathrm{q}}(S)=\iint_{S}\left(x-x_{\mathrm{c}}(S)\right)^{\mathrm{p}}\left(y-y_{\mathrm{c}}(S)\right)^{\mathrm{q}} \mathrm{d} x \mathrm{~d} y
$$

for $p, q \in\{0,1,2, \ldots\}$ and since it is supposed that the origin is placed at the centroid, we obtain

$$
\begin{aligned}
I(\varphi, S)= & (\sin \varphi)^{2} \bar{m}_{2,0}(S)-\sin 2 \varphi \cdot \bar{m}_{1,1}(S)+ \\
& (\cos \varphi)^{2} \bar{m}_{0,2}(S) .
\end{aligned}
$$

The minimum of the function $I(\varphi, S)$ is calculated in the following manner. The first derivative of the function $I(\varphi, S)$ is found

$$
\begin{aligned}
I^{\prime}(\varphi, S)= & 2 \sin \varphi \cos \varphi \cdot \bar{m}_{2,0}(S)-2 \cos 2 \varphi \cdot \bar{m}_{1,1}(S)- \\
& 2 \sin \varphi \cos \varphi \cdot \bar{m}_{0,2}(S),
\end{aligned}
$$


and then the first derivative is set to zero:

$$
\begin{aligned}
I^{\prime}(\varphi, S)= & \sin 2 \varphi\left(\bar{m}_{2,0}(S)-\bar{m}_{0,2}(S)\right)- \\
& 2 \cos 2 \varphi \cdot \bar{m}_{1,1}(S)=0 .
\end{aligned}
$$

From here, the required angle $\varphi$, but also the angle $\varphi+\pi / 2$, satisfies the equation

$$
\frac{\sin 2 \varphi}{\cos 2 \varphi}=\frac{2 \bar{m}_{1,1}(S)}{\bar{m}_{2,0}(S)-\bar{m}_{0,2}(S)} .
$$

By using trigonometric identities:

$$
\begin{gathered}
\sin ^{2} \alpha=\frac{1-\cos 2 \alpha}{2}, \cos ^{2} \alpha=\frac{1+\cos 2 \alpha}{2}, \\
\sin 2 \alpha=\frac{ \pm t g 2 \alpha}{\sqrt{1+\operatorname{tg}^{2} 2 \alpha}}, \cos 2 \alpha=\frac{ \pm 1}{\sqrt{1+\operatorname{tg}^{2} 2 \alpha}}
\end{gathered}
$$

and equation (3), the maximum and minimum of the function $I(\varphi, S)$ are obtained:

$$
\begin{gathered}
\max \{I(\varphi, S) \mid \varphi \in[0,2 \pi]\}=\frac{\bar{m}_{2,0}(S)+\bar{m}_{0,2}(S)}{2}+ \\
\frac{\sqrt{4\left(\bar{m}_{1,1}(S)\right)^{2}+\left(\bar{m}_{2,0}(S)-\bar{m}_{0,2}(S)\right)^{2}}}{2}
\end{gathered}
$$

$\min \{I(\varphi, S) \mid \varphi \in[0,2 \pi]\}=\frac{\bar{m}_{2,0}(S)+\bar{m}_{0,2}(S)}{2}-$

$$
\frac{\sqrt{4\left(\bar{m}_{1,1}(S)\right)^{2}+\left(\bar{m}_{2,0}(S)-\bar{m}_{0,2}(S)\right)^{2}}}{2} .
$$

The elongation of the shape $S$ is obtained as a quotient $\max _{\varphi \in[0,2 \pi)} I(\varphi, S)$ and $\min _{\varphi \in[0,2 \pi)} I(\varphi, S)$

$$
\varepsilon(S)=\frac{\max \{I(\varphi, S) \mid \varphi \in[0,2 \pi]\}}{\min \{I(\varphi, S) \mid \varphi \in[0,2 \pi]\}}
$$

The central geometric moments $\bar{m}_{\mathrm{p}, \mathrm{q}}(S)$ in the digital objects were replaced by the so-called central discrete moments $\bar{\mu}_{\mathrm{p}, \mathrm{q}}(S)$ defined by

$$
\bar{\mu}_{\mathrm{p}, \mathrm{q}}(S)=\sum_{(i, j) \in S \cap Z^{2}}\left(i-x_{\mathrm{cd}}(S)\right)^{\mathrm{p}}\left(j-y_{\mathrm{cd}}(S)\right)^{\mathrm{q}}
$$

where a real shape $S$ is represented with its digitization $\operatorname{dig}(S)$, while pixel $(i, j)$ belongs to $\operatorname{dig}(S)$ and the centroid of the discrete shape $S \cap Z^{2}$ is given by

$\left(x_{\mathrm{cd}}(S), y_{\mathrm{cd}}(S)\right)=\left(\frac{\sum_{(x, y) \in S \cap Z^{2}} x}{\sum_{(x, y) \in S \cap Z^{2}} 1}, \frac{\sum_{(x, y) \in S \cap Z^{2}} y}{\sum_{(x, y) \in S \cap Z^{2}} 1}\right)$.

Thus, the method for calculating the shape elongation is derived from the standard definition of the shape orientation. This procedure is simple in both versions, real and discrete, and the shape elongation measure is based on a simple calculus and standard algebraic operations, and it is easy to implement.

\subsection{Convexity measure}

The convexity is important shape descriptor in image processing and human vision. Here, convexity is defined as the ratio between shape's area and area of the its convex hull [28]:

$$
C_{\mathrm{x}}(S)=\frac{A(S)}{A(C h(S))}
$$

where $A(S)$ represents the shape's area and $A(C h(S))$ is the area of the minimum convex boundary circumscribing the shape $S$. It is illustrated in Fig. 1.

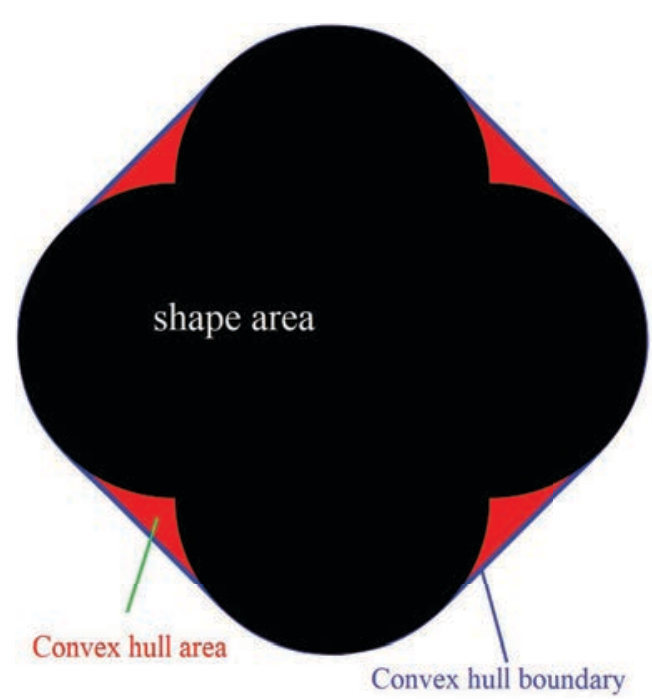

Fig. 1. The shape area and the convex hull area

The measure $C_{\mathrm{x}}(S)$ is an invariant with respect to translation, rotation and scaling. The convexity measure has the following desirable properties:

- $C_{\mathrm{x}}(S) \in(0,1]$,

- $C_{\mathrm{x}}(S)=1$ if and only if the measured shape $S$ is convex,

- there are shapes whose estimated convexity is arbitrarily close to 0 .

\subsection{Circularity measure}

The circularity is one of the most studied shape descriptors. It is usually defined by the following formula $C_{\text {st }}(S)=4 \pi A / P^{2}$, where $A$ represents shape's area while $P$ represents its perimeter. The disadvantages of this standard measure are discussed in the literature. Therefore, here we use the other one definition of the circularity based on the moments calculation defined by Zunic [31]:

$$
C(S)=\frac{\operatorname{Area}(S)^{2}}{2 \pi\left(\bar{m}_{2,0}(S)+\bar{m}_{0,2}(S)\right)}
$$

where $S$ is an arbitrary shape and $\operatorname{Area}(S)$ represents the shape's area. As with convexity, the measure $C(S)$ 
is an invariant with respect to translation, rotation and scaling. The circularity measure also has similar desirable features as well as a convexity measure:

- $C(S) \in(0,1]$,

- $C(S)=1$ if and only if the measured shape $S$ is circle,

- there are shapes whose estimated circularity is arbitrarily close to 0 .

\section{Results}

The morphology of the nanoparticle structures in TEM micrographs has been studied, Fig. 2(a) and Fig. 3(a), using proposed shape analysis (elongation, convexity and circularity).

In order to perform the shape analysis, the observed images should first be segmented. Fig.2(b) and Fig. 3(b) show Fig. 2(a) and Fig. 3(a) analyzed through R, G and $\mathrm{B}$ channels, respectively. If these images are watched through RGB histograms, it can be noticed that there are no significant oscillations between the observed channels, so the segmentation cannot be performed by analyzing the RGB channels. It is confirmed by the degree of matching by the structural similarity (SSIM) index which is based on the computation of three terms, namely the luminance term, the contrast term and the structural term. SSIM index shows large degree of matching. Namely, the SSIM values are from $96.27 \%$ to $98.80 \%$ depending on which two channels of Fig. 2(b) are compared, while these SSIM values are from $99.52 \%$ to $99.88 \%$ for Fig. 3(b).

It can be clearly seen that in the Figs. $2 \mathrm{a}$ and $3 \mathrm{a}$ the shade of gray are prevailing. In addition, brighter shades belong to areas outside the desired segments, ie nanoparticles. From the perspective of an 8-bit grayscale image, the pixel values of these brighter shades belonging obviously to the background. Such defined thresholds were applied to filtering of the corresponding images. The segmentation of Fig. 3(a) is somewhat more complicated because some nanoparticles overlap. It is taken into account that the segments are surrounded by brighter shades. The overlapping parts of the nanoparticles are processed by the method of parallel edge detection using differences in channel values for observed pixel and realized by standard command in MatLab. After that, the Sobel operator for edge detection is involved. The Sobel operator is based on the first derivative and it is technically a discrete differential operator used to calculate the approximation of the gradient of the image luminance function. The Sobel operator consists of two $3 \times 3$ matrices, which are transverse and longitudinal templates, and are plotted with the image plane, respectively, to obtain the difference between the horizontal and the longitudinal difference.

The images of isolated nanoparticles are shown in Fig. 2(c) (where nanoparticles are denoted by numbers from 1 to 2) and Fig. 3(c) (where nanoparticles are denoted by numbers from 1 to 13) which were then analyzed using the MatLab software package. Only nanoparticles that are shown in the picture as a whole are analyzed. The values of elongation, convexity and circularity for the two nanoparticles in Fig. 2(c) are shown in Table 1, while Table 2 shows the corresponding values of nanoparticles in Fig. 3(c).
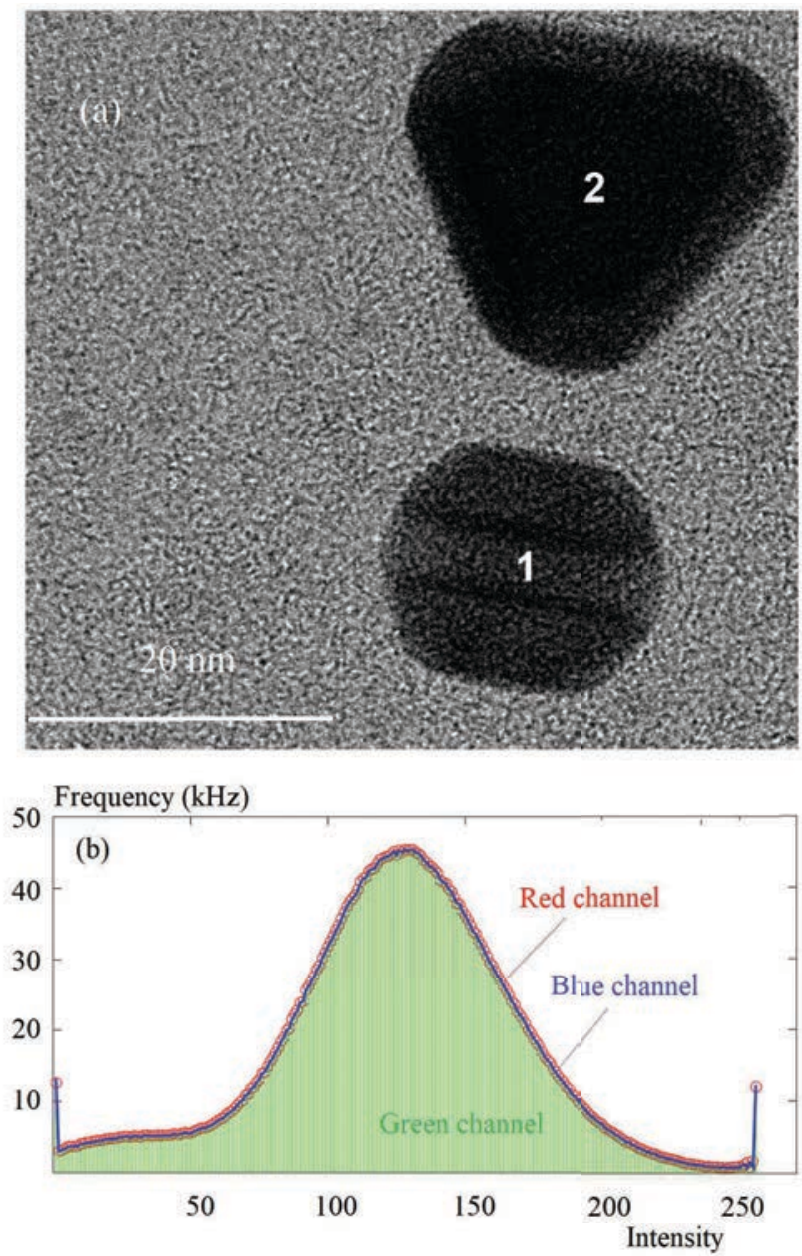

(c)

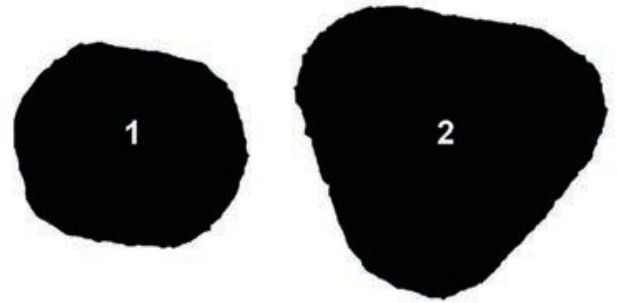

Fig. 2. (a) - TEM image of hematite, (b) - color histogram of (a), (c) - the two isolated nanoparticles from (a)

Table 1. Values of elongation, convexity and circularity of nanoparticles from Fig. 2

Nanoparticle, Elongation, Convexity, Circularity,

\begin{tabular}{lccc}
$S$ & $\varepsilon(S)$ & $C_{\mathrm{x}}(S)$ & $C(S)$ \\
\hline No. 1 & 1.3366 & 0.9847 & 0.9863 \\
No. 2 & 1.0872 & 0.9836 & 0.9665 \\
\hline
\end{tabular}



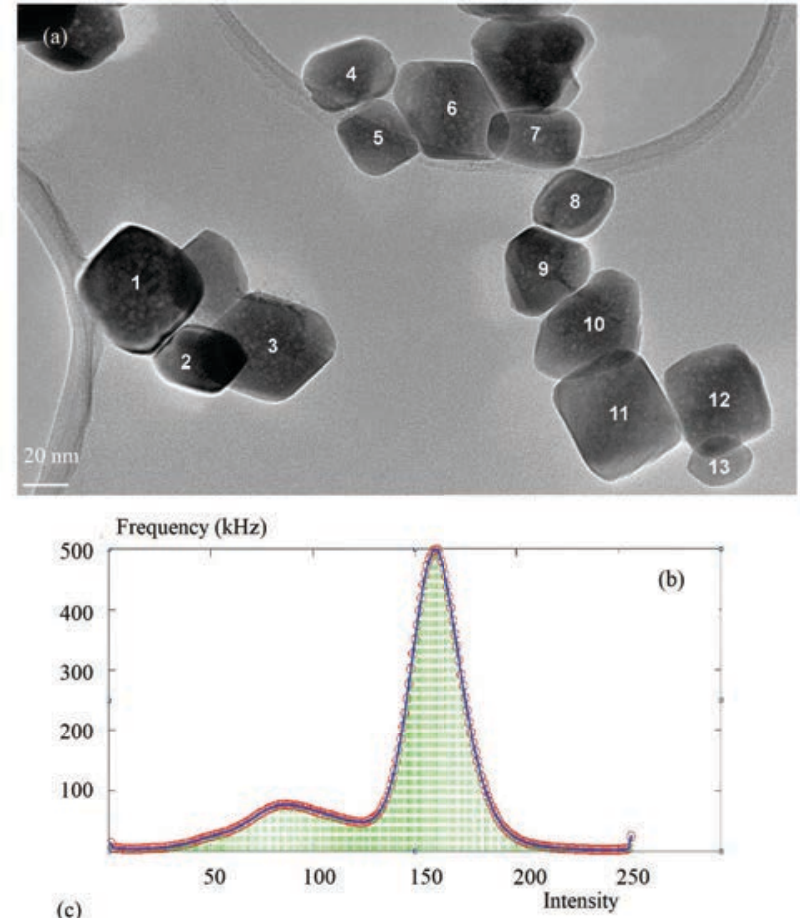

(c)

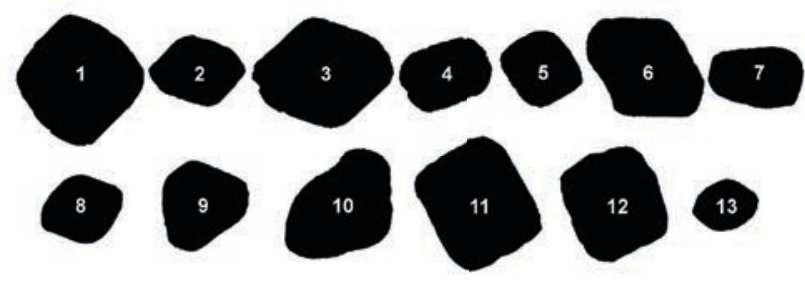

Fig. 3. (a) - TEM image of hematite, (b) - color histogram of (a), and (c) - the nanoparticles isolated from (a)

The literature states that no measure is good enough to describe all shapes. In support of this, we can point to the example of two nanoparticles in Fig. 2 which are different in shape, while the convexity measure for these nanoparticles gives approximately the same value (Ta- ble 1). The reason is that both nanoparticles are almost convex in shape and, thus, the values of the convexity are very close to 1 . Therefore, by the proposed convexity measure, we cannot distinguish these two (obviously different) shapes. On the other hand, the values of the circularity measure indicate that the nanoparticle No. 1 is slightly more circular than the nanoparticle No. 2, while the elongation measure in comparison with these two particles makes the biggest difference. Nanoparticle No. 1 is more elongated than No. 2 $(\varepsilon($ No.1 $)=1.3366 ; \varepsilon($ No.2 $)=1.0872)$. For these reasons, it is not enough to use only one measure, but for the more precise characterization of the shape it is proposed to use several different descriptors. The use of several different measures can contribute to a better understanding of the shape thanks to the quantitative characteristics obtained in this way.

Table 2. Values of elongation, convexity and circularity of nanoparticles from Fig. 3

\begin{tabular}{lccc} 
Nanoparticle, & $\begin{array}{c}\text { Elongation, } \\
S\end{array}$ & $\begin{array}{c}\text { Convexity, } \\
C_{\mathrm{x}}(S)\end{array}$ & $\begin{array}{c}\text { Circularity, } \\
C(S)\end{array}$ \\
\hline No. 1 & 1.0595 & 0.9903 & 0.9889 \\
No. 2 & 1.8561 & 0.9846 & 0.9445 \\
No. 3 & 1.7087 & 0.9871 & 0.9562 \\
No. 4 & 1.9927 & 0.9799 & 0.935 \\
No. 5 & 1.2742 & 0.9893 & 0.9821 \\
No. 6 & 1.928 & 0.9791 & 0.9363 \\
No. 7 & 2.4985 & 0.9884 & 0.8929 \\
No. 8 & 1.5411 & 0.9905 & 0.9681 \\
No. 9 & 1.2692 & 0.9877 & 0.9663 \\
No. 10 & 2.102 & 0.9785 & 0.9141 \\
No. 11 & 1.355 & 0.987 & 0.9697 \\
No. 12 & 1.2335 & 0.9847 & 0.9811 \\
No. 13 & 1.6678 & 0.9892 & 0.9649 \\
\hline
\end{tabular}

Fig. 3 is shown a wider range of the nanoparticle shapes. It is also indicated by the results of measures of elongation, convexity and circularity. The convexity

(a)

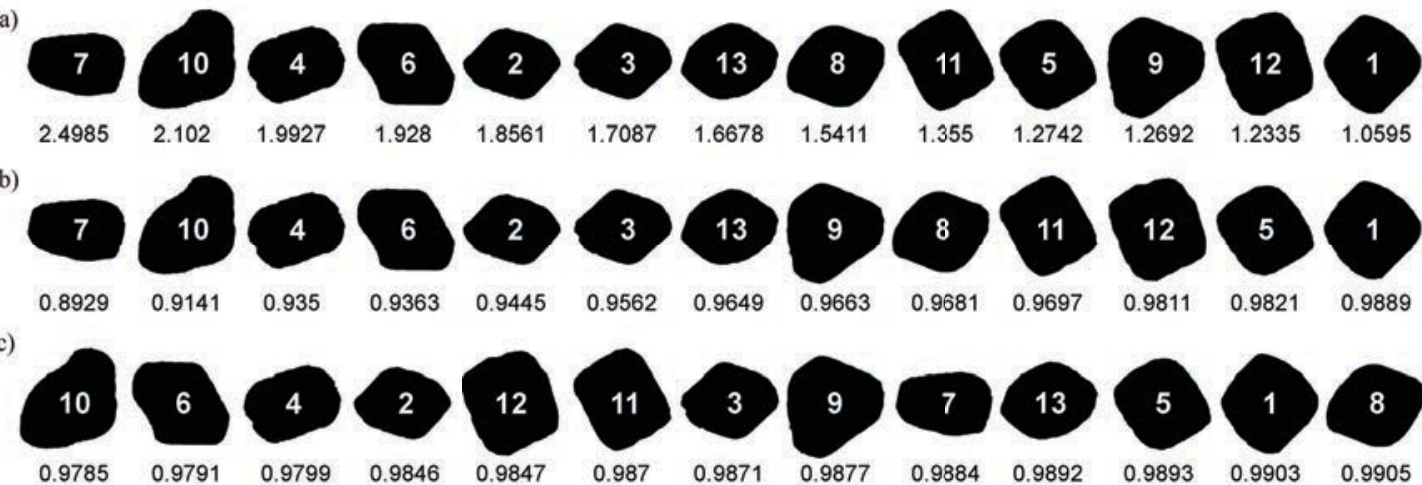

Fig. 4. Nanoparticles from Fig. 3(c) sorted by: (a) - elongation, (b) circularity, and (c) - convexity measure 
measure on this nanoparticle sample gives results in the range of 0.9785 (No. 10) to 0.9905 (No. 8). The convexity of the observed nanoparticles is quite high, and the least convex are nanoparticles No. 10 and No. 6. Observing Fig. 3(a), this can be interpreted as the influence of adjacent nanoparticles that have affected the convexity of its "neighbors". Namely, nanoparticle No. 5 is probably due to its tougher structure and proximity influenced the decrease of convexity No. 6, while No. 9 for the same reasons has influenced the decrease of the convexity of nanoparticle No. 10. On the other hand, the circularity measure gives values from the range of 0.8929 (No. 7) to 0.9889 (No. 1). It is visually clear that nanoparticles No. 7,10 , and 4 deviate most from the circular shape, which is confirmed by the quantitative results of the circularity measure. The proposed elongation measure $\varepsilon(S)$ in terms of the elongation of the nanoparticle shapes gives results that correspond to the visually expected. If all three proposed measures are observed, the elongation has the widest range of values of the observed sample (from 1.0595 to 2.4985 ). The most elongated nanoparticle is No. 7, while the least elongated No. 1.

In Fig. 4 the nanoparticles from Fig. 3(b) are sorted by the elongation, Fig. 4(a), circularity, Fig. 4(b) and convexity, Fig. 4(c) are shown. Since nanoparticles shape are analyzed, and their size does not affect the results of these measures, the nanoparticles are not shown in the original size, but are scaled to the same width.

The elongation and circularity measures give the same order for the first half of the series of nanoparticles from Fig. 4(a) and Fig. 4(b). The order of the first 7 nanoparticles is the same: $7,10,4,6,2,3,13$. However, in the second half of the series these two measures differently sort the nanoparticles from Fig. 3(c). This is in line with numerous studies dealing with the quantification of shapes that emphasize the need for the use of several different shape descriptors (circularity, elipticity, orientability) [15, $25,31]$. This is conditioned by the fact that no descriptor is sufficiently reliable to perform shape characterization solely on the basis of it.

\section{Conclusion}

In materials science, shape analysis requires research that should enable a more precise characterization of nanoparticles shape, and therefore a better understanding of their impact on the physical properties of the material. For the quantification of shapes, shape descriptors are used whose basic idea is the possibility of distinguishing of shapes. However, it is known that no descriptor is not good enough to be used in all situations, and the use of each shape descriptor has advantages and disadvantages. Therefore, for more precise determination of the nanoparticle shape, it is recommended to combine several different measures.

In this paper for the shape characterization of the nanoparticles, it is proposed to use the measures of elongation, convexity and circularity that are based on the calculation of discrete moments. The described measures are good candidates for the shape analysis of nanoparticles, due to the relatively simple realization and fast execution time of the algorithms. This article presents experimental results were obtained from TEM images of hematite nanoparticles $\left(\alpha-\mathrm{Fe}_{2} \mathrm{O}_{3}\right)$ from which individual nanoparticles were isolated and analyzed. The results show that the measures of elongation, convexity and circularity give intuitively expected results, which is a desirable feature. Thanks to these measures it is possible to improve the efficiency of nanoparticle classification in terms of estimate their shape. Besides this, these shape analysis algorithms can be used to understand the relationship between structure, morphology and magnetic properties of nanomaterials, as well as applications in other disciplines such as biomedicine, industry, geology, astronomy.

\section{Acknowledgements}

M. T. and L. K. acknowledge the financial support from the Ministry of Education and Science of the Republic of Serbia (Grant no. III 45015 and III 044006), Serbia-Slovakia bilateral project 2017-2018 (SKSR-2016-0055) and Serbia-Belarus 2018-2019 (451-03003036/2017-09/06). B. L. acknowledges financial support from the Ministry of Education and Science of the Republic of Serbia (Grant no. 171007).

\section{REFERENCES}

[1] G. Tong, Y. Liu, T. Wu, C. Tong, and F. Du, "" $\mathrm{H}_{2} \mathrm{O}$-steered size/phase evolution and magnetic properties of large-scale, monodisperse $\mathrm{Fe}_{\mathrm{x}} \mathrm{O}_{\mathrm{y}}$ nanomaterials", Journal of Materials Chemistry C, vol. 3, pp. 5506-5515, 2015.

[2] B. Ahmed, S. Kumar, S. Kumar and A. K. Ojha, "Shape induced (spherical, sheets and rods) optical and magnetic properties of $\mathrm{CdS}$ nanostructures with enhanced photocatalytic activity for photodegradation of methylene blue dye under ultra-violet irradiation", Journal of Alloys and Compounds, vol. 679, pp. 324-334, 2016.

[3] S. P. Gubin, Y. A. Koksharov, G. B. Khomutov and G. Y. E. Yurkov, "Magnetic nanoparticles: preparation, structure and properties", Russian Chemical Reviews, vol. 74, pp. 489-520, 2005.

[4] R. M. Fratila, S. Rivera-Fernández and M. Jesús, "Shape matters: synthesis and biomedical applications of high aspect ratio magnetic nanomaterials", Nanoscale, vol. 7, pp.8233-8260, 2015.

[5] H. Gavilán, O. Posth, L. K. Bogart, U. Steinhoff, L. Gutiérrez and M. P. Morales, "How shape and internal structure affect the magnetic properties of anisometric magnetite nanoparticles", Acta Materialia, vol. 125, pp. 416-424, 2017.

[6] A. H. Lu, E. E. Salabas and F. Schüth, "Magnetic nanoparticles: synthesis, protection, functionalization, and application", Angewandte Chemie International Edition, vol. 46, pp. 1222-1244, 2007.

[7] A. Nagata, H. Sato, Y. Matsui, T. Kaneko and Y. Fujiwara "Magnetic properties of carbon nanotubes filled with ferromagnetic metals", Vacuum, vol. 87, pp. 182-186, 2013.

[8] L. Kopanja, D. Žunić, B. Lončar, S. Gyergyek and M. Tadić "Quantifying shapes of nanoparticles using modified circularity and ellipticity measures", Measurement, vol. 92, pp. 252-263, 2016. 
[9] M. Mohammadikish, "Hydrothermal synthesis, characterization and optical properties of ellipsoid shape $\alpha$-Fe2O3 nanocrystals", Ceramics International, vol. 40, pp. 1351-1358, 2014.

[10] L. Kopanja, M. Tadić, S. Kralj and J. Žunić, "Shape and aspect ratio analysis of anisotropic magnetic nanochains based on TEM micrographs", Ceramics International, vol. 44, pp. 12340-12351, 2018.

[11] T. O. Paine, L. I. Mendelsohn and F. E. Luborsky, "Effect of shape anisotropy on the coercive force of elongated single-magnetic-domain iron particles", Physical Review, vol. 100, pp. 1055, 1995.

[12] Z. Wang, "A new approach for segmentation and quantification of cells or nanoparticles", IEEE Transactions on Industrial Informatics, vol. 12(3), pp. 962-971, 2016.

[13] Y. C. Chen, Y. C. Chen, Y. C. and A. S. Chiang, "Template-driven segmentation of confocal microscopy images", Computer methods and programs in biomedicine, vol. 89(3), pp. 239-247, 2008.

[14] L. Li and Q. An, "An in-depth study of tool wear monitoring technique based on image segmentation and texture analysis", Measurement, vol. 79, pp. 44-52, 2016.

[15] M. A. Akta and J. Žunić, "Measuring shape ellipticity", Computer Analysis of Images and Patterns, Springer, Berlin, Heidelberg, pp. 170-177, 2011.

[16] A. Boschetto and V. Giordano, "Powder sampling and characterization by digital image analysis", Measurement, vol. 45, pp. 1023-1038, 2012.

[17] E. S. Gadelmawla, "Simple and efficient algorithms for roundness evaluation from the coordinate measurement data", Measurement, vol. 43, pp. 223-235, 2010.

[18] A. M. Herrera-Navarro, H. Jiménez-Hernández and I. R. Terol-Villalobos, "Framework for characterizing circularity based on a probability distribution", Measurement, vol. 46, pp. 4232-4243, 2013.

[19] M. Ilić, I. Budak, M. V. Vasić, A. Nagode, U. Kozmidis-Luburić, J. Hodolič and T. Puškar, "Size and shape particle analysis by applying image analysis and laser diffraction-Inhalable dust in a dental laboratory", Measurement, vol. 66, pp. 109-117, 2015.
[20] R. Klette and J. Žunić, "Digital approximation of moments of convex regions", Graphical Models and Image Processing, vol. 61, pp. 274-298, 1999.

[21] P. L. Rosin, "Measuring shape: ellipticity, rectangularity, and triangularity", Machine Vision and Applications, vol. 14, pp. 172-184, 2003.

[22] P. L. Rosin and J. Žunić, "Measuring squareness and orientation of shapes", Journal of Mathematical Imaging and Vision, vol. 39, pp. 13-27, 2011.

[23] A. B. Sekuler, "Axis of elongation can determine reference frames for object perception", Canadian Journal of Experimental Psychology, vol. 50, pp. 270, 1996.

[24] M. A. Taylor, "Quantitative measures for shape and size of particles", Powder Technology, vol. 124, pp. 94-100, 2002.

[25] J. Zunic, P. L. Rosin and L. Kopanja, "On the orientability of shapes", IEEE Transactions on Image Processing, vol. 15, pp. 3478-3487, 2006.

[26] J. Žunić, L. Kopanja and J. E. Fieldsend, "Notes on shape orientation where the standard method does not work", Pattern Recognition, vol. 39, pp. 856-865, 2006.

[27] J. Žunić, R. Kakarala and M. A. Aktas, "Notes on shape based tools for treating the objects ellipticity issues", Pattern Recognition, vol. 69, pp. 141-149, 2017.

[28] J. Zunic and P. L. Rosin, "A new convexity measure for polygons", IEEE Transactions on Pattern Analysis and Machine Intelligence, vol. 26, pp. 923-934, 2004.

[29] R. Mukundan and K. R. Ramakrishnan, Moment functions in image analysis-theory and applications, World Scientific, 1998.

[30] M. Stojmenović and J. Žunić, "Measuring elongation from shape boundary", Journal of Mathematical Imaging and Vision, vol. 30, pp. 73-85, 2008.

[31] J. Žunić, K. Hirota and P. L. Rosin, "A Hu moment invariant as a shape circularity measure", Pattern Recognition, vol. 43, pp. $47-57,2010$.

Received 19 March 2019 